\title{
DIE SCHWEIZERISCHE KULTURLANDSCHAFT ZUR ZEIT JOHANN STUMPFS
}

\author{
Dem Gedächtnis des Erscheinens der Schweizer Chronik 1547 gewidmet
}

\author{
Von ERNST WiNKLER
}

Mit 3 Abbildungen

Vor nunmehr 400 Jahren erschien «bey Christoffel Froschower, Zürych», die "Gemeiner Loblicher Eydgnoschafft Stetten, Landen und Völckeren Chronickwirdiger thaaten beschreybung» von JoHANN STUMPF aus Bruchsal (Baden), Dekan von Oberwetzikon und Stein a. Rh., Reformator und Freund ZwINGLIS, von Zeitgenossen und Nachwelt Livius Helvetiae genannt ${ }^{1}$. Das nahezu 1700 Großquartseiten, an die 2400 Holzschnitte und 23 Landkarten enthaltende Werk umriß erstmals umfassend nicht nur die Geschichte, sondern auch die Landeskunde der Eidgenossenschaft im Rahmen ihrer Umwelt, die um so unmittelbarer auf uns wirkt, als ihr Verfasser nicht nur Quellen verwertet, sondern viele Gebiete erwandert hatte. So darf es als einer der wesentlichen Ausgangspunkte auch der schweizerischen Kulturlandschaftsgeschichte betrachtet werden. Zwar hat die spätere wissenschaftliche Kritik in zunftgerechter Akribie zahlreiche Haare in dem monumentalen Gericht entdeckt und objektiv zergliedert ${ }^{2}$. Eines konnte sie der Leistung nicht vorenthalten: Sie mußte ihr, nicht zuletzt infolge ihrer Gesamtschweiz und Teilregionen zum Ausdruck bringenden "Landtaflen ${ }^{3}$ zuerkennen, daß sie ein Denkmal beginnenden eidgenössischen Bewußtseins sei, wie es einprägsamer kaum ein anderes Werk der damaligen Zeit darstellte. Es rechtfertigt sich deshalb wohl, seiner in Form eines knappen Rekonstruktionsversuches der damaligen Landschaft auch von der Geographie aus zu gedenken, wobei vor allem STUMPF selbst das Wort erteilt wird.

Die Zeichen, unter denen die Eidgenossenschaft um die erste Hälfte des 16. Jahrhunderts stand, sind ja auch für unsere Epoche in mehrfacher Beziehung der Erinnerung würdig. Wie jetzt, hatte die Welt eine säkulare Gärung ergriffen (Reformation - Gegenreformation) und unser Land sich zu folgenschweren Entscheiden zu entschließen. Es war die Zeit des Erwachens des Neutralitätsgedankens. Zudem war die Schweiz, räumlich betrachtet, auf der Höhe ihrer Macht angelangt, so daß auch ihre Physiognomie besondere Aspekte bot. Denn die "Dreizehn Orte" umfaßten mit Zugewandten und Herrschaften gut die Fläche der heutigen Eidgenossenschaft ${ }^{4}$. Auf diesem Areal wohnten um 1550 nach der sorgfältigen Schätzung $H$. AmmanNs 0,8 Millionen Menschen ${ }^{5}$. Die mittlere Dichte betrug 17 Einwohner pro $\mathrm{km}^{2}$, wobei über 20 auf das Mittelland entfielen. Es besa $\beta$ damit mehr als die doppelte Dichte von Jura und Alpen. Dem Eidgenossen des 16. Jahrhunderts standen also im Durchschnitt 5-6 ha Boden zur Verfügung (1941:1), und es muß erstaunen, wenn der blühende Reislauf jener Zeit (auch) aus den mangelnden Erwerbsmöglichkeiten zu erklären versucht wurde. Immerhin schlossen der damalige Stand der Technik und die relativ niedere Bevölkerungszahl großräumige

1 Zitiert nach dem im Staatsarchiv Zürich enthaltenen Erstdruck.

${ }^{2}$ H. Müller: Der Geschichtsschreiber JohanN Stumpr. Eine Untersuchung seines Weltbildes. Zürich 1945.

3 Die Kartenausschnitte aus Stumprs Chronik verdanken wir dem Geographischen Verlag Kümmerly \& Frey in Bern, der in verdienstlicher Weise zusammen mit Leo WeIsz den StumpFschen Atlas 1942 neu herausgab und dadurch auch den kurzen Rückblick anregte.

4 K. MEYER: Geographische Voraussetzungen der eidgenössischen Territorialbildung. Schwyz 1927.

5 H. Ammann: Die Bevölkerung der Westschweiz im ausgehenden Mittelalter. Festschrift Welti, S. 390-447. Aarau 1937. 


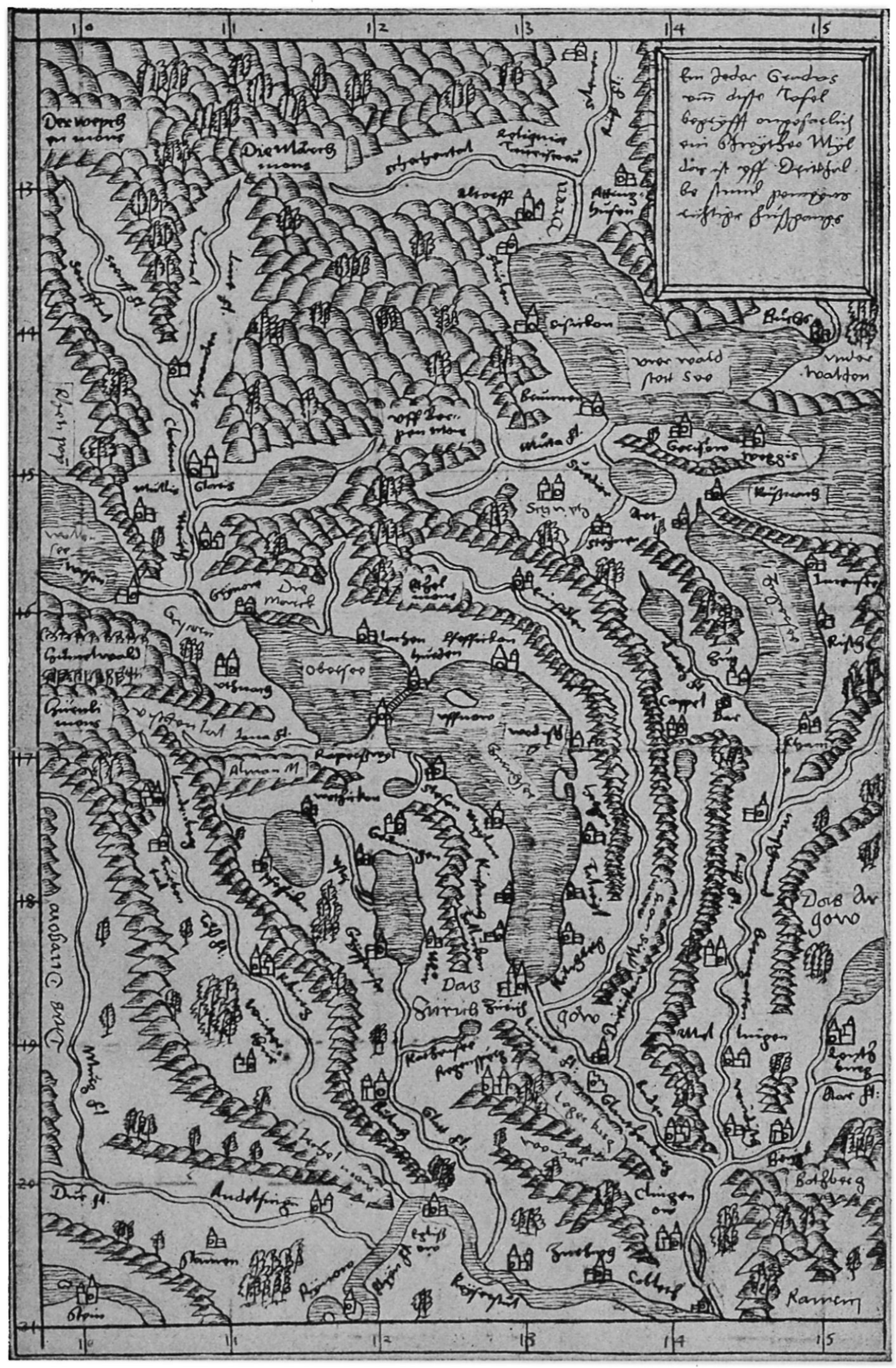

«Das Zürich gouw»

Entwurf StumpFs, teilweise nach eignen Itineraraufnahmen 
Meliorationen und Gewässerkorrektionen aus ${ }^{6}$, so daß eine erheblich stärker von Naturrisiken Überschwemmungen, Lawinen, Dürren usw. - beherrschte, extensiver genutzte und dünner besiedelte Kulturlandschaft im ganzen wie im einzelnen bestand, als wie sie sich heute präsentiert.

Ihre Hauptelemente bildeten außer den zumeist noch ungebändigt fließenden Flüssen und Seen, den noch ausgedehnten Sümpfen und dem wohl bereits bis zur jetzigen Verteilung gelangten Wald Höfe, Weiler, Dörfer, Kleinstädte, Klöster und Burgen inmitten von Dreifelder- und Weidefluren, wobei, wie schon $H$. BERNHARD ${ }^{7}$ betont hat, das Siedlungsmaschennetz in den Grundzügen dem gegenwärtigen entsprach. Das Zahlenverhältnis der Land- zu den Stadtbewohnern stand nach AMmanN etwa 4:1. Doch handelte es sich bei den städtischen Siedlungskomplexen um Größenordnungen, deren obere Grenze 10000 Einwohner waren (Genf; die nächstgrößern: Bern, Freiburg, Lausanne, Zürich hatten höchstens 5000$)^{8}$. Im einzelnen gestaltete sich die Organisation der Landschaft kaum weniger komplex als in der Gegenwart. Das tritt aus StUMPFs Darstellung ebenso eindrücklich hervor, wie es die spätere Detailforschung bestätigte. Die Gliederung der Landschaft bestimmte, von den Marken der Natur abgesehen, vor allem die Einteilung in Gemeinde- und Privatland. Ersteres zerfiel in Wald, Ried und Weide, letzteres in Saat- (Sommer- und Wintersaat) und Brachland und war schon im 15. und 16. Jahrhundert stark parzelliert. Die nähere Umgebung der Siedlungen kennzeichneten Krautund Baumgärten, Hanf- und Flachpünten und Roosen, kleine, oft künstliche Teiche zur Beize der Gespinstpflanzen; große Areale besaßen auch die Reben, gegen deren Überhandnehmen schon im 15. Jahrhundert eingeschritten wurde. In ausgesprochenem Maße war die Schweiz eine Bocagelandschaft, deren zahlreiche grüne und hölzerne Latten- und Bretterzäune sowohl in den Acker- als in den Grasbaugebieten das Antlitz der meisten Gegenden anmutig belebten. Die wildreichen Wälder mußten schon vielerorts gegen Übernutzung durch Weide und Holzentzug geschützt werden. Andrerseits bestanden im Jura und Alpenvorland noch urwüchsige Waldgebiete, in die erst zögernd die Rodung vordrang. So boten Feld- und Waldfluren das Bild einer wohl großenteils erschlossenen, aber kaum systematisch gepflegten Agrarlandschaft, die jedoch im Begriffe stand, industrialisiert zu werden (Refugianten). Wie sie trugen Siedlung und Verkehr patriarchalische Züge. Das Wegenetz, im einzelnen kaum weniger verzweigt als heute, war nach Reiseberichten und Vorschriften primitiv ausgebaut und der damals immerhin bedeutende interne und externe Verkehr stark behindert. Der Schiffahrt kam eine bedeutsame Rolle zu. Zwischen Städten und Dörfern, die sich vor allem durch die Umwallung und engere Überbauung der erstern unterschieden, bestanden viele Übereinstimmungen. Sie gelangten, von den Haustypen abgesehen, der weitgehenden Selbstversorgungswirtschaft der Zeit gemäß in zahlreichen Mühlen, Obst- und Oltrotten, Hanf- und Flachsreiben, Lohstampfen und Schleifen zum Ausdruck. Zu ihnen bildeten die unvermeidlichen Einrichtungen der Rechtsexekutive (Pranger und Galgen) merkwürdige Kontraste. Im Rückgang begriffen waren Feudalsiedlungen, Burgen, Schlösser, wie auch die Klöster, demgegenüber teils prunkhafte Bürgerhäuser herrschend wurden.

Diesen teilweise zwiespältigen Erscheinungen stellt STUMpF ein erheblich idealeres Bild seiner Zeit gegenüber. Obwohl auch eir damalige rohe Sitten, Zwistigkeiten und Reislauf brandmarkt, erscheint seine "Eidgnoschafft" doch im ganzen als ein Land der Prosperität. Dies kommt in den folgenden Ausschnitten aus der Gesamtschilderung und der Darstellung einer Landschaft zum Ausdruck.

«Die landschafft der Helvetier ist an merteils orten vonn art wild rauch und bergig, besonders gegen dem Alpgebirg ... (aber) sölich gantz land (hat) sein alte wilde gestalt also gar verwandlet ... dann da sind gemeinlich alle berg und täler bey unseren tagen gesübert, erbauwen, bewonet und über die maßen lieblich und fruchtbar, also daß diß land yetz hat weyns und allerhand getreid und guter früchten ein gute notturfft: ist auch an allerley nutzbaren vych gar reych, hat deßhalb nit allein an fleisch, käß, milch, schmaltz, ziger, honig, unnd allerley lieblichem obs, sunder auch an visch, wildprät und gefügel ein überfluß ... Die allerhöchsten Alpen und gebirg (die doch vor augen der ansähenden allein velsen unnd schnee erzeigend) sind innwendig, so man sy ersteyget, voll schöner wisen und schöner graßreycher weyden, auß denen über die maß vil vychs erneert und erzogen wirt. Also daß Helvetia an vych, fleisch, milch, schmaltz, käß etc. nit allein für sich selbs überflüssig reych, sonder auch mit söliche wahr andre anstoßenden landen träffentlich dienstlich und beholffen ist: dan söliche wahr wirt in die umligenden Italische, Gallische und Teutsche lender gefürt. Das tregt groß gelt un gut, dann sölichs die tägliche erfarnuß leert, namlich, daß welcher vermag 20 kuy

6 Für vereinzelte Versuche vgl. Rhein- und Glattakten (1447-1797) des Staatsarchivs Zürich sowie E. WinkLeR: Zur Geschichte der Glattkorrektionen. Neue Zürcher Zeitung 156, 1935, Nr. 1820.

$2 \mathrm{H}$. Bernhard: Die betriebswirtschaftlichen Verhältnisse (der zürcherischen Landwirtschaft). Die Landwirtschaft im Kanton Zürich, S. 59-123. Zürich 1924.

8 H. Ammann: a. a. O. 
summer und winter zeerhalten, dem ertraget sy järlich ob 100 Kronen nutzung über allen kosten den er mit den knechten tregt: geschwigen der kalber unnd aufwachs des jungen vychs so darvon erzogen wirt. Und söliche wahr wirt alle geradtsamet und erobert mit geringer arbeit, darumb ist auch $\mathrm{di} ß$ land reych an leuten, groß volck erneert sich in kleiner gegne (Gegend). Wenig bättler auß Helvetien findr man in andern landen, aber Helvetia laufft all zeyt voll frömder armen, das mich offt gewundert hat wär sy alle speyse und erhalte. Aber dise Helvetia ist auß Gottes sägen fruchtbar... hat alles das en guet fruchtbar erdtrich tragen sol, ire gebirg stäckend voll metall und ertz, des doch wenig gesucht wirt. Auch erregend sich ... gar mancherley heilsamer brunnen, wasser unnd beder, warm und kalt von deren yedem besonder an seinem ort gesagt wirt ...

Tacitus schreybt, daß die alten Teutschen wenig stett, und ire wonungen oder heuser weyt voneinander gesetzt und abgesundert habind, nach dem eines yeden hof, gut, gewerb, brunne, holtz ... erfordert. Diser brauch des bauwens ist . . . bey den Helvetijs noch gantz gemein ... kompt nit auß mangel der kunst, oder unwüssenheit des bauwens, sonder werded die gebeuw also... weyt von einanderen gesetzt, fheur und brunst ze verhüten (dann ire heuser merteils von holtz gemachet, und mit holtz oder strauw gedeckt sind ...) oder das ein yeder sein wonung auff seine gut haben möge: dann dieweyl diß land aller tyranney entladen ist ... wie fürnäm und gschickt die ... Eydgnossen im zimbern . . . ärtiger gebeuwen diser zeyt syend, des gebend ire zierliche und wolgebauwte stett gute kundtschafft. Welcher stetten... Helvetia in irem alten kreiß hat an der zal ob 70 außgenommen all ire pundtsgenossen, unnd die bey unseren zeyten ire ringmauren haben lassen zerfallen; und sich dennocht jrer alten stattsatzungen unnd rechtungen noch gebrauchend.

Alle stett, fläcken, straaßen und tabernen ligend voll kauffleut, voll frömds weyns, voll außlendischer geschläck, gewürtz und frömder wahr. Es ist yetz in Helvetia nit mer wol geläbt, wo man nit seltzame Welsche trachten und essen fürtregt: dahär sy zum wolläben also entbrünned, das vil von völle und des leybs feißte zum krieg und allen arbeitsamen übungen untüchtig werdend. Die alten gebrauchtend sich schlächter bekleidung, jre tücher machtend sy selbs, etliche halb lyne, halb wulle, etliche gar wullin und grob... Aber diser zeyt ist kein land ... der Eydgnoschafft in kostlicheir und kleideren zevergleychen. Gold, Silber, Sammat und Seyden, ist bey allen stende ein gmeine tracht, daryn bringed die kriegsleut alle zeyt etwas neuwer sitten auß frömbden kriegen und auch gewonlich etwas neuwer plaagen und lasteren ... Die Priester sind... in eeren gehalten ... Der Adel wirt auch für andere stand geeret, habend jre eigene herrschafften, sitz, Schlösser, Gericht, Titel etc., doch dörffend sy kein krieg füren, kein tyranney treyben und müssend auch selbs yedem ansprechigen recht geben und nemen vor der oberkeit der statt oder lands darunter sy gesässen sind. Die Burgerschafft der stetten erneerend sich eins teils allerhand kauffmannsgwerben, die anderen durch allerley handwerck: etlich behelffend sich der güteren. Die Landleut Helvetiae habend dreyerley gwerb, etlich den Ackerbauw, und das ist der gröste teil: die anderen bauwend den weyn: die dritten, deren auch gar vil ist, umb alle gebirg erneerend sich allein des vychs, des sy so vil habend, das nit die weyber allein, sonder starcke menner und knecht die küy melckend, käß und ziger machend. Die werdend genennt Sennen, jre wonungen und werckstatt Sennhütten...

(Wie die ganze Eidgenossenschaft ist auch das) Wallis eine gar herrliche landschafft... ligt ob den Helvetiern zwüschend den höchsten Alpgebirg, ein eng talgelend, hat aber beiderseyts fruchtbare berg, auch vil nebendtäler... is gerings herumb... umbzogen und beschlossen mit wunderhohen und grausamen gebirgen, die sich merteils auff ein gute Teutsche meyl hoch gegen den wulcken und lüfften aufrichtend, also das derselbigen bergen vil zue allen zeyten stätigklich mit Glettscher, Firn oder schnee bedeckt sind ... deßhalb man gemeinlich an allen orten, wo man dareyn oder darauß 


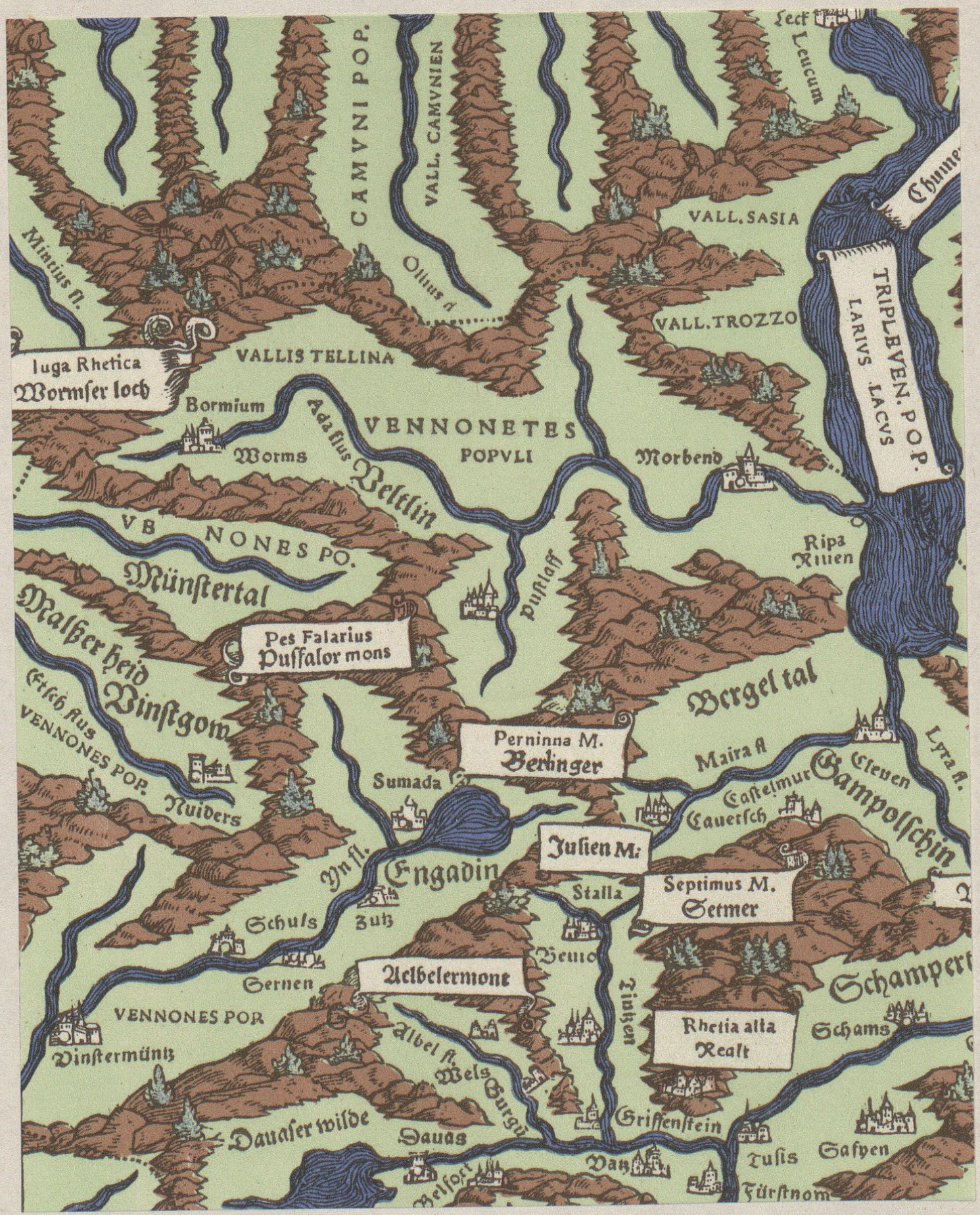

Kartenausschnitt aus "DIE LANDTAFELN DES JOHANN STUMPF“

Kümmerly \& Frey . Geographischer Verlag . Bern 


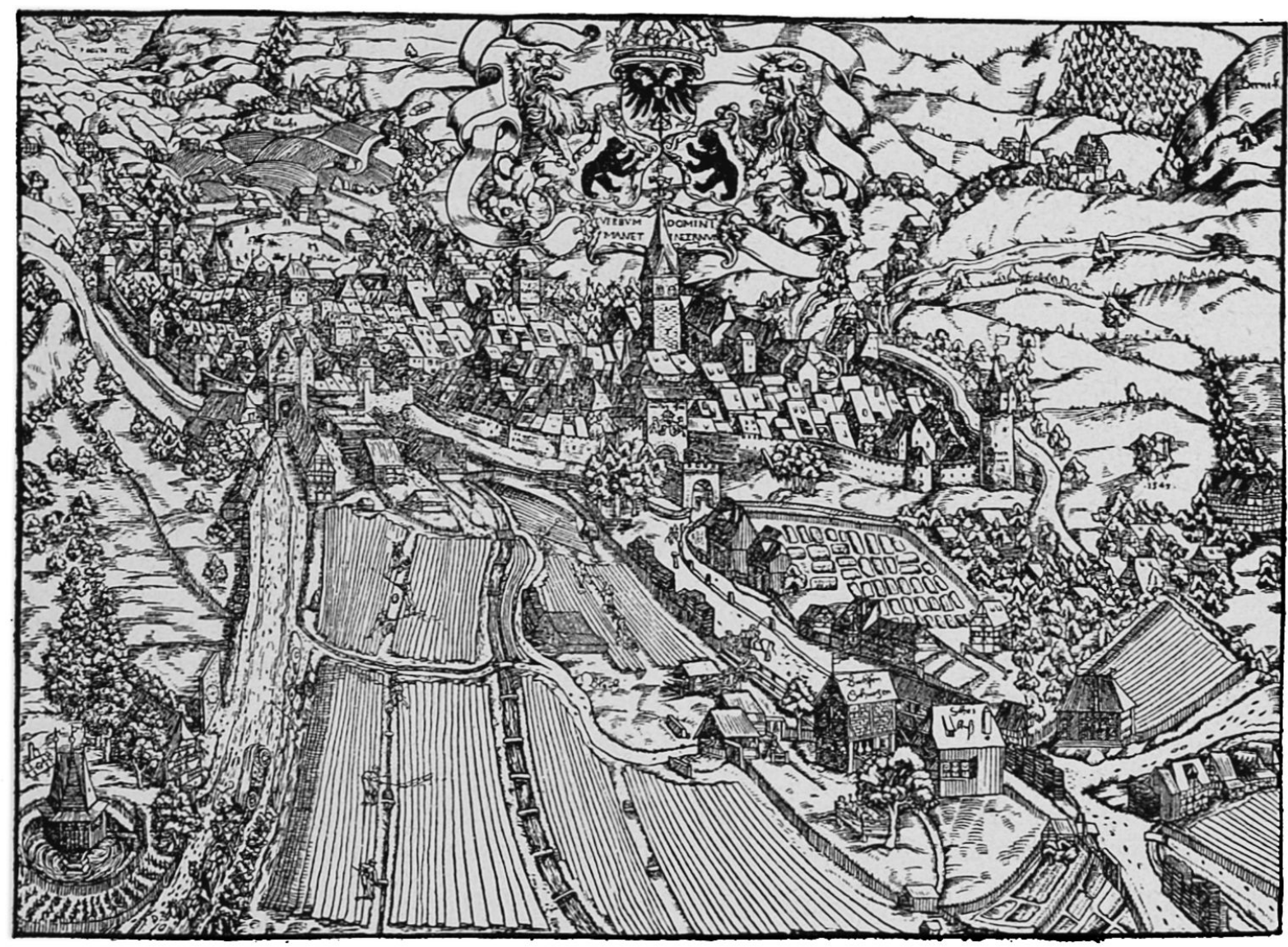

St. Gallen 1545. Eines der geographisch instruktivsten Bildbeispiele aus Stumprs Chronik. Im Mittelgrund die umwallte Stadt, vorn ihr «Industriequartier», die Bleichen und Schießanlage, sowie ländliche Block- und Riegelbauten. Im Hintergrund die Wiesenflur und der Bodensee

wandlen wil, hohe berg, rauhe velsen und gefärliche wäg ersteygen muß: dann gemeinlich alle päßz und eyngeng jres lands von natur und höhe des wilden gebirgs also wunderbarlich bevestiget sind, das sy durch kleine macht beschirmt... möchtind werden ... Wjewol nun das land Wallis mit dem allerhöchsten ... Schneegebirg ... umbzogen, ist es doch im talgelend auß der maßen fruchtbar und so lieblich, dergleychen ich nit acht ein so fruchtbar land in so wildem gebirg unter der Sonnen erfunden werden, das schaffet, daß es gar in die Sonnen gericht ist, erstreckt sich von Aufgang gegen Nidergang, hat die Sonn den gantzen tag, dardurch es also gefruchtbaret ist, daß in disem land keiner dingen zu menschlichem gebrauch ... mangel ist: dann da wachsend allerley guter und Edler früchten, weyn, korn, haber, schmalsadt, obs und hat darzu an visch, fleisch und wildprät ein überflußz.

Der weynwach $ß$ erhebt sich ob dem Zehenden Brick zu Möril und gadt durchs land nider biß zue S. Mauritzen, nimpt ye lenger ye reychlicher zue. Es hat weyß und rot weyn mancherley art ... In den Zehen Leuck, Syder und Sitten hat der rot weyn den preyß vor dem weyßen, der wirt also schwartz und dick, daß man darmit schreyben möcht ... Die weyngewächs dises lands sind also lieblich, starck und gut, als man sy in Teutschen landen sölte finden ... Es wirt auch zu gmeinen jaren dises gueten weyns vil über das gebirg gesomet... in andere land, als gen Uri, gen Haßle, gen Sanen, inns Sibental, Fruetingertal und biß gen Bern etc.

Allerley getreid hat diß land gnueg, als Weytzen, Rogken, Gersten. Das underland hat mer Winterfrücht... und das ober mer Summerfrücht. Das erdtrich ist gantz fruchtbar, also das auch zu oberist im land im Zehenden Goms die äcker gemeinlich 
alle jar frucht gebend ... An vilen orten wässerend sy alle jre güter, richtend das wasser auch etwan durch jre äcker und weyngärten und könnend dasselbig gar artig an den bergen här leiten durch gräben und kännel. Es hat auch im land eigne rechtung und breuich umb die wässerung der güeter... Das gantz land is durchpflantzet mit allerley Obs und gueter Böumfrüchten, als öpffel, biren, nußz, pflumen, allerhand heimischer und wilder kirschen, auch kestinen, mulbeer, pfersich, haselnußz ... söliche gemeine frücht hat es auch umb Sitten, Syder und Gundiß, Mandel, Feygen, Granaten und dergleychen Edler früchten, die auch sunst in den allerfruchtbaristen Teutschen landen nicht gmeinlich im brauch sind. Darzue erzeucht man ... vil guets Saffran.

Die gebirg... habend gemeinlich schöne wäld und weiden, jr gmein holtz ist Lerchen, Arben, weyB und rote Tannen. $\mathrm{Da}$ findt man auch weyße und rote Cristallen, besser und größer dann bey den Lepontiern. So hat das land vil Ertz und Bergwerck ... Under dem Wildprät sind allein Hirtzen, Recher und wilde Schweyn dem land Wallis ungemein und seltzsam (selten), sunst hat es ... ein völlige gnüege aller der thieren: als Bären, Wölff, Thierwölff, Lüchs, Füchs, Marder ... und allerley gefügels ... Fasanen, Parnyssen, Haselhüener, Steinhüener, Räbhüener etc. Das wildprät von Steinböcken, Ybschgeißen (Steingeißen) und Gembsen ist in disem land also gemein, $\mathrm{da}$ man söliches.bey weylen in der Metzg nebend anderem gemeinem fleisch außhauwt und umb ein gering gelt verkauff ... Das land hat allerley heimischer thieren ... als Roßz, maulthier, esel, schweyn, pfauwen, genß, enten, hüner, küngelin und tauben ... darzu erneert es vil ymben oder Bynle ... Des Rindervychs ... Item Schaaff und geißen etc. hat dis land ein überflußz ... Der flußz Rodan ... ertragend allerley visch ... Von den warmen und heilsamer Bedern ... wird ich hernach schreyben ...."

In ähnlicher Weise wird des damals offenbar regen Steinkohlen- und Erzbergbaus, der Tal- und Alpwirtschaft und schließlich der Siedlungen und des Walliservolks gedacht, das StuMPF als fleißig und klug schildert, wobei er nicht nur das Land als Ganzes, sondern auch seine Landschaften gesondert würdigt. Es wird hieraus eindrücklich, da $B$ das Wallis offensichtlich schon im 16. Jahrhundert eine kulturlandschaftliche Oase einzigartigen Gepräges gebildet hat. Wenn auch den meisten übrigen Gebieten der damaligen Eidgenossenschaft das Lob fruchtbarer Landschaften zuerkannt wird, tritt das Große Tal doch als Sonderfall aus ihnen heraus. Deshalb wurde es hier auch als Beispiel Srumprscher Landschaftsbeschreibung gewählt.

So tritt uns aus der STUMPFschen Chronik das Gesamtgefüge einer schweizerischen Kulturlandschaft des 16 . Jahrhunderts entgegen, das dank der allmählich sich vertiefenden Neutralitätspolitik seiner Staatsmänner trotz innerer Zwistigkeiten die Ausgangslage einer zukunftsreichen Entwicklung bildete. Ihr ein anspornendes Symbol geschaffen zu haben, ist nicht zuletzt Verdienst des Zürcher Pfarrers JohanN STuspf, dem wir deshalb als Eidgenossen wie als Heimatkundler auch heute Dank und, was mehr ist, Nachachtung schulden.

\section{LE PAYSAGE CULTUREL SUISSE AU TEMPS DE JOHANN STUMPF}

A l'occasion du centenaire de la Chronique suisse de 1547, l'auteur esquisse la situation gtographique de la Suisse au $16 e$ siocle. Ses caractères fondamentaux etraient la petite ville et le village, les champs de culture a triple assolement et les pàturages. A l'évolution politique correspondait le début d'une transformation du paysage, qui se manifestait par l'augmentation du nombre des habitations, de la population et l'amélioration de l'économic.

\section{IL PAESAGgio CULTURALE SVIZZERO AL TEMPO DI JOHANN STUMPF}

In memoria della cronaca svizzera del 1547 vien schizzata in situazione geografica della Svizzera nel 16. secolo. I suoi caratteri fondamentali erano il borgo $c$ il villaggio, la campicoltura a rotazione e l'erbicoltura con sfruttamento comune ed inoltre un progressivo sviluppo del transito intemazionale. All'evoluzione politica corrisponde un frazionamento del paesaggio culturale (aumento del numero delle abitazioni, ecc.) che si manifesta nei suoi stadi iniziali. 